\title{
Acute Shock in an Ambiguous Child
}

Chanchal Das ${ }^{1}$, Pranab Kumar Sahana ${ }^{2}$, Nilanjan Sengupta ${ }^{3}$, Mukut Roy $^{4}$, Deshmukh Ashish $^{5}$, Ranen Dasgupta ${ }^{6}$

\begin{abstract}
Patients with congenital adrenal hyperplasia (CAH) usually presents with varied manifestations. In female, it can manifest as ambiguous genitalia, salt wasting crisis or androgen excess in puberty depending on the severity of enzyme deficiency. Here, we report a four and half year old girl who developed salt wasting crisis in the neonatal period. Prompt diagnosis and immediate glucocorticoid and fludrocortisone replacement saved her life. High index of suspicion is needed to diagnose CAH and continued replacement of glucocorticoid and mineralocorticoid is needed to suppress virilization of the female child and prevent further crises.
\end{abstract}

Key Words: Congenital adrenal hyperplasia, Ambiguous genitalia, Salt wasting crisis, Glucocorticoid, Fludrocortisone.

\section{Introduction}

Congenital Adrenal Hyperplasia (CAH) comprises a group of autosomal recessive disorders caused by deficient adrenal corticosteroid biosynthesis. It results from defects in one of the steroidogenic enzymes involved in cortisol biosynthesis. Between $90 \%$ and $95 \%$ of cases of $\mathrm{CAH}$ are caused by 21 $\alpha$-hydroxylase deficiency. ${ }^{1}$ Among several distinct clinical varieties of $\mathrm{CAH}$, non-classic form is the most common. In female, salt-wasting form ( 1 in 20,000 live births) usually presents with sexual ambiguity at birth and develop salt wasting crisis later part in the neonatal period. We report an interesting case of acute adrenal crisis in the neonatal period.

\section{Case Report}

A four-and-half-year-old girl attended our endocrine department for the evaluation of sexual ambiguity. Past medical history revealed that the girl was born in a non consanguineous family with an average birth weight without any significant antenatal history. It was a full term, normal vaginal home delivery. Immediate post-natal period was uneventful. Though she had sexual ambiguity at birth, the parents did not seek any medical advice. After third week of age, the baby had developed lethargy, poor

1 Chanchal, Das, M.B.B.S (Cal), DCH (Cal), MD (Medicine), Post Doctoral Trainee (Endocrinology)

2 Pranab Kumar Sahana, MD (Medicine), DM (Endocrinology), Associate Professor

3 Nilanjan Sengupta, MD (Medicine), DM (Endocrinology), Associate Professor \& Head

4 Mukut Roy, M.B.B.S, MD (Medicine), Post Doctoral Trainee (Endocrinology)

5 Ashish Deshmukh, M.B.B.S, MD (Medicine), Post Doctoral Trainee (Endocrinology)

6 Ranen Dasgupta, MD (Medicine), DM (Endocrinology), Professor \& Ex-Head Department of Endocrinology, N.R.S. Medical College \& Hospital, Kolkata-700014, West Bengal, India.

Corresponding Author:

Dr. Chanchal Das,

Department of Endocrinology, N.R.S. Medical College \& Hospital, 138, A.J.C.Bose Road,

Kolkata-700014, West Bengal, India

Email: drchanchal.das@gmail.com feeding, vomiting, decreased cry and activity. On the $31^{\text {st }}$ day of her life, she was admitted in neonatal intensive care unit in a gasping condition. On examination, the baby had features of shock with hypothermia, and altered mental status. Examination of external genitalia revealed enlarged clitoris (clitoral Index-150 $\mathrm{mm}^{2}$ ), complete labial fusion (Prader stage 3), hyperpigmentation of labio-scrotal folds and urethral meatus just beneath the clitorophalus (Figure 1).

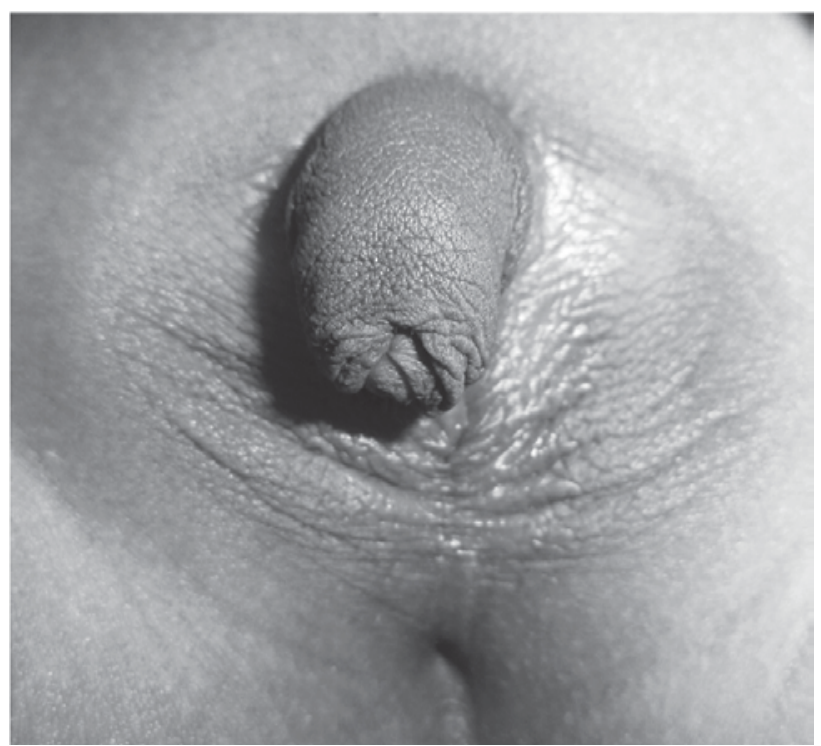

Figure 1: Showing clitoromegally, fusion of labia causing ambuity in a female child

There were no signs of sepsis clinically or biochemically. All biochemical reports were normal except hyponatremia [121 meq/L (135-145)], hypokalemia [3.1 meq/L (3.5-4.5)] and hypoglycaemia (62 mg/dl). Serum 17-Hydroxy-Progesterone (17 OH-P) and Dehydroepiandrostenedion - Sulphate (DHEA-S) were high [> $2000 \mathrm{ng} / \mathrm{dL}(3-8)$ and $3.9 \mu \mathrm{g} / \mathrm{mL}$ (0.9-1.8) respectively] and undetectable cortisol $(<1.0 \mu \mathrm{g} / \mathrm{dl})$ and elevated adrenocorticotropin hormone (ACTH) [109 $\mathrm{pg} / \mathrm{mL}$ (9-52)]. Ultrasonography of abdomen showed bilateral enlarged adrenal glands (Figure 2, 3). 


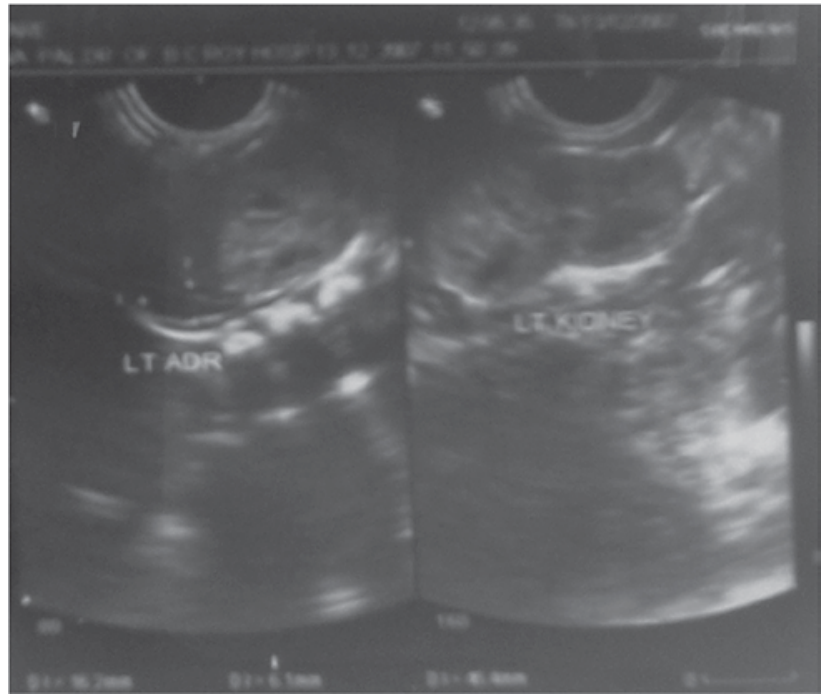

Figure 2: Ultrasonography of pelvis showing enlarged left adrenal gland (16 mm $\times 6 \mathrm{~mm}$, age 7 month)

Presence of Mullerian duct and absence of any Wolfian duct structures (Figure 4). With the classical features of salt wasting, absence of sepsis, female sex and sexual ambiguity at birth, the baby was diagnosed as a case of salt wasting form of CAH (SW-CAH). Sample was sent for Karyotyping which later showed 46, XX.

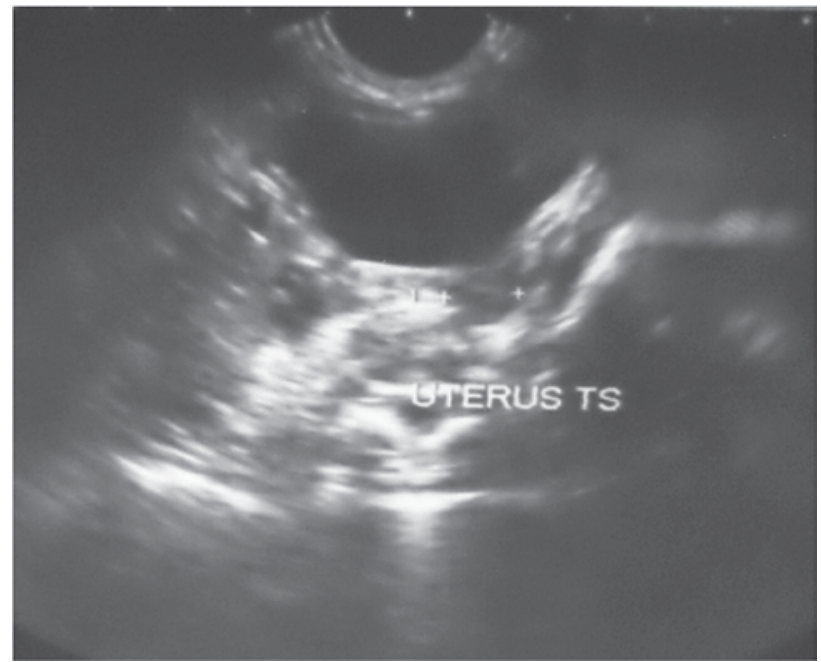

Figure 4: Ultrasonography of pelvis showing the presence of uterus (age 7 month)

The girl was resuscitated and given intravenous hydrocortisone and fludrocortisone. Her electrolytes became normal on the $7^{\text {th }}$ day. At present she is being followed up with oral hydrocortisone and fludrocortisone. Her height and weight are $94 \mathrm{~cm}\left(<3^{\text {rd }}\right.$ percentile; Ht-SDS: -2.02$)$ and 15 kilograms $\left(>25^{\text {th }}\right.$ but $<50^{\text {th }}$ percentile) respectively. Her blood pressure is normal $(72 / 44 \mathrm{mmHg})$. She is still having features of virilization in the form of enlarged clitorophalus. Therefore, paediatric surgeon has been consulted for consideration of clitoroplasty and vaginoplasty which would be done very soon.

\section{Discussion}

The incidence of $\mathrm{CAH}$ due 21- $\alpha$-hydroxylase deficiency varies from 1 in 10,000 to 1 in 15,000 live births ${ }^{2}$, but in isolated communities due to consanguinity it may be much higher (1: 300 in Alaskan Inuit populations). In our case there is no history of consanguinity and negative family history of similar disorder. The condition arises because of defective conversion of 17- $\alpha$-OHP to 11-deoxycortisol. Reduced cortisol biosynthesis results in reduced negative feedback drive and increased ACTH secretion. ${ }^{3}$ Seventy-five percent of patients have clinically manifest mineralocorticoid deficiency because of failure to convert sufficient progesterone to deoxycorticosterone (DOC). We found hyponatremia in our case due to deficiency of mineralocorticoids. Hypokalemia is probably due to vomiting otherwise hyperkalemia would have been present. The enhanced ACTH drive to adrenal androgen secretion in utero leads to virilization of an affected female fetus. Depending on the severity, clitoral enlargement, labial fusion, and development of a urogenital sinus may occur, leading to sexual ambiguity at birth and even inappropriate sex assignment. ${ }^{4}$ Neonates commonly present after the first 2 weeks of life with a salt-wasting crisis and hypotension due to exhaustion of serum cortisol derived from mother as the activity of the affected enzyme is zero no cortisol is being synthesized in the baby. Our case also had typical features of virilisation described above and presented in the later part of neonatal period, consistent with the literature. ${ }^{5}$ The clinical signs and symptoms of salt wasting include poor feeding, vomiting, failure to thrive, lethargy, and sepsis-like symptoms. This baby also presented with similar types of features. These features may alert the clinician to the diagnosis in a male baby, but the diagnosis is still delayed in many cases, and the condition carries a significant neonatal mortality rate. The overall treatment goal is to replace glucocorticoid and mineralocorticoid, thereby preventing further salt-wasting crises, but also to normalize adrenal androgen secretion so that normal growth and skeletal maturation can proceed ${ }^{6}$ and virilisation is prevented. ${ }^{7}$

\section{Conclusion}

Salt wasting form of Congenital Adrenal Hyperplasia is an uncommon clinical entity. High index of suspicion is needed to diagnose this condition. Early recognition and prompt treatment with glucocorticoid and mineralocorticoid is essential to save the newborn.

\section{References :}

1. White PC, Speiser PW. Congenital adrenal hyperplasia due to 21-hydroxylase deficiency. Endocr Rev. 2000; 21:245-291.

2. Paul MS, Nils PK. Adrenal cortex. Williums text book of endocrinology $12^{\text {th }}$ Ed. S. Melmed et. al., (Saunders 2011): 482-580.

3. Merke DP, Bornstein SR. Congenital adrenal hyperplasia. Lancet. $2005 ; 365: 2125-2136$

4. Rocha RO, Billerbeck AE, Pinto EM, et al. The degree of external genitalia virilization in girls with 21-hydroxylase deficiency appears to be influenced by the CAG repeats in the androgen receptor gene. Clin Endocrinol (Oxf). 2008; 68:226-232.

5. Joint LWPES/ESPE CAH Working Group. Consensus statement on 21-hydroxylase deficiency from the Lawson Wilkins Pediatric Endocrine Society and the European Society for Paediatric Endocrinology. J Clin Endocrinol Metab. 2002; 87:4048-4053.

6. Eugster EA, Dimeglio LA, Wright JC, et al. Height outcome in congenital adrenal hyperplasia caused by 21-hydroxylase deficiency: a metaanalysis. J Pediatr. 2001; 138:26-32.

7. Hindmarsh PC. Management of the child with congenital adrenal hyperplasia. Best Pract Res Clin Endocrinol Metab. 2009; 23: $193-208$. 